\title{
A New Hydroxyjasmone Glucoside and Its Related Compounds from the Leaf of Thyme
}

\author{
Junichi Kitajima, ${ }^{*}$ Toru Ishikawa, and Atushi Urabe \\ Showa Pharmaceutical University; Higashi-Tamagawagakuen 3, Machida, Tokyo 194-8543, Japan. \\ Received April 5, 2004; accepted June 2, 2004
}

\begin{abstract}
From the polar portion of the methanol extract of thyme (leaf of Thymus vulgaris; Labiatae), which has been used as a stomachic, carminative, a component of prepared cough tea, and as a spice, a new hydroxyjasmone glucoside, $(Z)-5^{\prime}$-hydroxyjasmone $5^{\prime}-O$ - $\beta$-D-glucopyranoside was isolated together with five related compounds and four aromatic compounds. The structures of the new compound was clarified by spectral investigation.
\end{abstract}

Key words thyme; Thymus vulgaris; hydroxyjasmone; $5^{\prime}$-hydroxyjasmone $5^{\prime}$ - $O$ - $\beta$-D-glucopyranoside; $(1 R, 2 R)$-methyl-5'-hydroxyjasmonate

The leaf of thyme [Thymus vulgaris; Labiatae] is a most popular herbal medicine and spice used world wide. It is listed in German and British Herbal Pharmacopoeia, and has been used as a stomachic, carminative, diuretic, urinary disinfectant, and vermifuge. Thyme is indigenous to central and southern Europe, and is now widely cultivated as a tea which promotes rest and relaxation. ${ }^{1)}$ Most of the studies on this leaf were concerned with the essential oil containing mainly the isomeric monoterpenoid thymol $(30-70 \%)$ and carvacrol (3-15\%), and other monoterpenoids such as methylcarvacrol, $p$-cymene, $\alpha$-phellandrene, limonene, $\alpha$-terpineol, terpinen-4-ol, 1,8-cineole, borneol, bornyl acetate and so on. ${ }^{2,3)}$ However, no report had been published on the polar portion of this leaf. Therefore, we undertook investigation of the polar portion of this leaf, in continuation of our studies on the polar constituents of spices and herbal medicines. ${ }^{4}$ In this paper, we discuss the compounds related to hydroxyjasmonic acid derivative, which are interesting for their metabolic formation and defense responses in higher plants. ${ }^{5,6)}$

The commercial thyme leaf was extracted with methanol, and the methanolic extract was suspended in water and successively extracted with ether and ethyl acetate. The aqueous layer was chromatographed on Amberlite XAD-II to give water and methanol eluate fractions, respectively. The methanol eluate fraction was chromatographed on Sephadex LH-20, and subjected to a combination of silica gel, Lobar RP-8 column chromatography and HPLC to isolate six compounds related to hydroxyjasmone $(\mathbf{1}-\mathbf{6})$, three aromatic glucosides, 3-hydroxy-4-methoxyphenethyl 3-O- $\beta$-D-glucopyranoside (7), ${ }^{7)}$ eugenol $\beta$-D-glucopyranoside $(\mathbf{8}){ }^{8}{ }^{8}$ syringin (9), ${ }^{9)}$ and rosmarinic acid (10). ${ }^{10,11)}$ On the other hand, five glucides (glycerol, D-hamamelose, D-fructose, D-glucose and sucrose) were isolated from the water eluate fraction. Among them, $\mathbf{4}$ is a new compound.

Glucoside $1\left(\mathrm{C}_{12} \mathrm{H}_{22} \mathrm{O}_{6}\right.$, an amorphous powder, $[\alpha]_{\mathrm{D}}^{23}$ $-31^{\circ}$ ) was identified as $(Z)$-3-hexenyl 1- $O$ - $\beta$-D-glucopyranoside, which was isolated from the leaf of Pertya glabrescens. ${ }^{12)}$ Recently, aglycone of $\mathbf{1}$ found to induce the expression of defense genes in uninfected leaves. ${ }^{13)}$

Glucosides $2\left(\mathrm{C}_{18} \mathrm{H}_{28} \mathrm{O}_{9}\right.$, an amorphous powder, $[\alpha]_{D}^{24}$ $\left.-72^{\circ}\right)$ and $3\left(\mathrm{C}_{19} \mathrm{H}_{30} \mathrm{O}_{9}\right.$, an amorphous powder, $\left.[\alpha]_{\mathrm{D}}^{21}-55^{\circ}\right)$ were identified as $(1 R, 2 R)-5^{\prime}$-hydroxyjasmonic acid $5^{\prime}-O-\beta$ D-glucopyranoside, which was isolated from the leaf of Perilla frutescens, ${ }^{14)}$ and $(1 R, 2 R)$-methyl-5'-hydroxyjasmonate $5^{\prime}$ - $O-\beta$-D-glucopyranoside, which was isolated as a potato tuberforming substance from Jerusalem Artichoke, ${ }^{15)}$ respectively.

Glucoside $4\left(\mathrm{C}_{17} \mathrm{H}_{26} \mathrm{O}_{7}\right.$, an amorphous powder, $[\alpha]_{D}^{22}$ $\left.-24^{\circ}\right)$ showed $[\mathrm{M}+\mathrm{Na}]^{+},[\mathrm{M}+\mathrm{H}]^{+}$and $\left[\mathrm{M}+\mathrm{H}-\mathrm{C}_{6} \mathrm{H}_{12} \mathrm{O}_{6}\right]^{+}$ ion peaks at $\mathrm{m} / \mathrm{z} 365,343$ and 163 in their positive ion FABMS, and its molecular formula was suggested from the accurate mass number of the $[\mathrm{M}+\mathrm{H}]^{+}$and $[\mathrm{M}+\mathrm{Na}]^{+}$ion peak in the high-resolution positive ion FAB-MS. Acid hydrolysis of 4 gave glucose, and its ${ }^{1} \mathrm{H}-,{ }^{13} \mathrm{C}-\mathrm{NMR}$ and ${ }^{1} \mathrm{H}-{ }^{13} \mathrm{C}$ COSY spectral data (see Experimental) showed the presence of one tert-methyl, four methylenes, one hydroxylated methylene, one disubstituted double bond, one tetrasubstituted double bond, and one carbonyl group, in addition to the $\beta$-glucopyranosyl moiety. ${ }^{16,17)}$ From comparison of the NMR spectral data with those of $\mathbf{1}$ to $\mathbf{3}$, and analysis of its HMBC spectral data (see Experimental), the aglycone of $\mathbf{4}$ was found to be $5^{\prime}$-hydroxyjasmone and the location of the glucosyl group was $\mathrm{C}-5^{\prime}$. The glucose was suggested to be D-form by comparison of its $[M]_{\mathrm{D}}$ value $\left(-82^{\circ}\right)$ with those of $\mathbf{1}\left(-81^{\circ}\right)$ and methyl $\beta$-D-glucopyranoside $\left(-62^{\circ}\right){ }^{18)}$ The pentenyl double bond was determined to be $Z$ by its coupling constant between $\mathrm{H}-2^{\prime}$ and $\mathrm{H}-3^{\prime}(J=10.5 \mathrm{~Hz})$. So, 4 was characterized as (Z)-5'-hydroxyjasmone $5^{\prime}$ - $O$ - $\beta$-D-glucopyranoside

Compound $5\left(\mathrm{C}_{12} \mathrm{H}_{18} \mathrm{O}_{4}\right.$, an amorphous powder, $[\alpha]_{D}^{24}$ $\left.-67^{\circ}\right)$ was identified as $(1 R, 2 R)-5^{\prime}$-hydroxyjasmonic acid first isolated from the culture filtrate of the tropical fungus Botryodiplodia theobromae, ${ }^{19)}$ and $\mathbf{6}\left(\mathrm{C}_{13} \mathrm{H}_{20} \mathrm{O}_{4}\right.$, an amor-
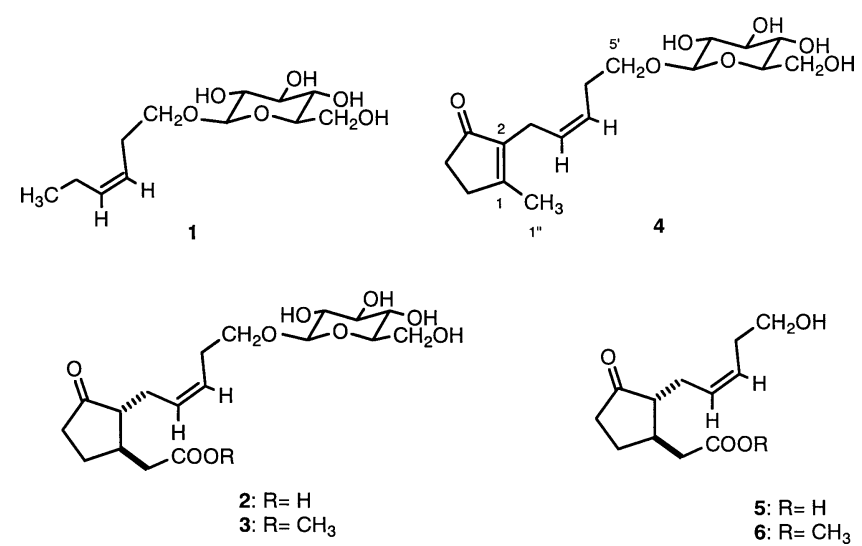

Fig. 1. Structures of $\mathbf{1}-\mathbf{6}$ 
phous powder, $\left.[\alpha]_{\mathrm{D}}^{21}-49^{\circ}\right)$ was identified as $(1 R, 2 R)$ methyl-5'-hydroxyjasmonate, which was derived by Kramell et $a l^{20)}$

The aglycone of $\mathbf{4}$ and compound $\mathbf{6}$ were reported for the first time from the plant.

\section{Experimental}

Melting points were determined on a Yanagimoto micromelting point apparatus and are uncorrected. Optical rotations were measured on a JASCO DIP-370 digital polarimeter. FAB-MS were recorded with a JEOL HX-110 spectrometer using glycerol as matrix. ${ }^{1} \mathrm{H}$ - and ${ }^{13} \mathrm{C}-\mathrm{NMR}$ spectra were taken on a JEOL A-500 spectrometer with tetramethylsilane as an internal standard, and chemical shifts were recorded in $\delta$ value. ${ }^{1} \mathrm{H}-{ }^{13} \mathrm{C}$ COSY, HMBC spectra were obtained with standard JEOL software. Column chromatography (C.C.) was carried out under TLC monitoring by using Kieselgel 60 (70-230 mesh, Merck), Sephadex LH-20 (25-100 $\mu \mathrm{m}$, Pharmacia), Lobar RP-8 column (Merck) and Amberlite XAD-II (Organo). TLC was performed on silica gel (Merck 5721) and spots were detected with $p$-anisaldehyde$\mathrm{H}_{2} \mathrm{SO}_{4}$ reagent. HPLC separation was carried out with Symmetryprep $\mathrm{C}_{18}$ $7 \mu \mathrm{m}$ [Waters; column size, $7.8 \times 300 \mathrm{~mm}$; ODS] and carbohydrate analysis [Waters; column size, $3.9 \times 300 \mathrm{~mm}$; CHA] columns.

Extraction and Separation Commercial thyme leaves (the leaf of Thymus vulgaris L.; purchased from Asaoka Spices Ltd., Lot. No. 00011801; $2.0 \mathrm{~kg}$ ) were extracted with methanol $(51 \times 2)$ for 2 weeks, and the extract $(419.1 \mathrm{~g})$ was partitioned berween ether/water. An aqueous phase was extracted with ethyl acetate. Removal of the solvents from each phase gave ether $(140.3 \mathrm{~g})$, ethyl acetate $(20.9 \mathrm{~g})$ and aqueous $(257.9 \mathrm{~g})$ fractions. The aqueous fraction was chromatographed over Amberlite XAD-II $\left(\mathrm{H}_{2} \mathrm{O} \rightarrow \mathrm{MeOH}\right)$ to give water eluate $(106.6 \mathrm{~g})$ and methanol eluate $(143.4 \mathrm{~g})$ fractions. The methanol eluate fraction was subjected to Sephadex LH-20 $(\mathrm{MeOH})$ to give five fractions (frs. A-E). Fraction B (124.3 g) was chromatographed over silica gel $\left[\mathrm{CHCl}_{3}-\mathrm{MeOH}-\mathrm{H}_{2} \mathrm{O}(17: 3: 0.2 \rightarrow 4: 1: 0.1 \rightarrow\right.$ $7: 3: 0.5) \rightarrow \mathrm{MeOH}]$ to give 13 fractions (frs. $\mathrm{B}_{1}-\mathrm{B}_{13}$ ). Fraction $\mathrm{B}_{3}(6.7 \mathrm{~g}$ ) was passed through a Lobar RP-8 column $\left[\mathrm{MeCN}-\mathrm{H}_{2} \mathrm{O}(3: 17 \rightarrow 3: 7)\right]$ to give 25 fractions (frs. $\mathrm{B}_{3-1}-\mathrm{B}_{3-25}$ ), and fr. $\mathrm{B}_{3-8}$ was subjected to HPLC [CHA, $\left.\mathrm{MeOH}-\mathrm{H}_{2} \mathrm{O}(19: 1)\right]$ to give $\mathbf{1}(9 \mathrm{mg})$ and $\mathbf{4}(23 \mathrm{mg})$. Fraction $\mathrm{B}_{3-19}$ was subjected to HPLC [ODS, MeCN- $\mathrm{H}_{2} \mathrm{O}(1: 1)$ ] to give $6(2 \mathrm{mg})$ and 8 $(51 \mathrm{mg})$. Fraction $\mathrm{B}_{4}(5.5 \mathrm{~g})$ was passed through a Lobar RP-8 column [MeCN- $\left.\mathrm{H}_{2} \mathrm{O}(3: 17 \rightarrow 3: 7)\right]$ to give 21 fractions (frs. $\mathrm{B}_{4-1}-\mathrm{B}_{4-21}$, and fr. $\mathrm{B}_{4-2}$ was subjected to silica gel column chromatography $\left[\mathrm{CHCl}_{3}-\mathrm{MeOH}-\mathrm{H}_{2} \mathrm{O}\right.$ $(9: 1: 0.1)$ ] to give $5(34 \mathrm{mg})$. Fraction $\mathrm{B}_{4-9}$ was subjected to HPLC [CHA, $\left.\mathrm{MeCN}-\mathrm{H}_{2} \mathrm{O}(49: 1)\right]$ to give $3(23 \mathrm{mg})$. Fraction $\mathrm{B}_{5}(4.3 \mathrm{~g})$ was passed through a Lobar RP-8 column $\left[\mathrm{MeCN}-\mathrm{H}_{2} \mathrm{O}(3: 17 \rightarrow 3: 7)\right]$ to give 20 fractions (frs. $\mathrm{B}_{5-1}-\mathrm{B}_{5-20}$ ), and fr. $\mathrm{B}_{5-4}$ was subjected to HPLC [ODS, $\mathrm{MeCN}-\mathrm{H}_{2} \mathrm{O}(1: 3)$ ] to give $9(10 \mathrm{mg})$. Fraction $\mathrm{B}_{6}(5.8 \mathrm{~g})$ was passed through a Lobar RP-8 column $\left[\mathrm{MeCN}-\mathrm{H}_{2} \mathrm{O}(3: 17)\right.$ to give 15 fractions (frs. $\mathrm{B}_{6-1}-\mathrm{B}_{6-15}$ ), and fr. $\mathrm{B}_{6-5}$ was subjected to HPLC [CHA, MeCN- $\mathrm{H}_{2} \mathrm{O}(19: 1)$ ] to give $7(46 \mathrm{mg})$. Fraction $\mathrm{B}_{7}(1.2 \mathrm{~g})$ was passed through a Lobar RP-8 column $\left[\mathrm{MeCN}-\mathrm{H}_{2} \mathrm{O}(3: 17 \rightarrow 3: 7)\right]$ to give 10 fractions (frs. $\left.\mathrm{B}_{7-1}-\mathrm{B}_{7-10}\right)$, and fr. $\mathrm{B}_{7-5}$ was subjected to silica gel column chromatography $\left[\mathrm{CHCl}_{3}-\mathrm{MeOH}-\mathrm{H}_{2} \mathrm{O}(7: 3: 0.5)\right]$ to give $2(11 \mathrm{mg})$. Fraction D $(7.6 \mathrm{~g})$ was chromatographed over silica gel $\left[\mathrm{CHCl}_{3}-\mathrm{MeOH}(3: 2) \rightarrow \mathrm{MeOH}\right]$ to give $\mathbf{1 0}$ $(1.5 \mathrm{~g})$. A part of the water eluate fraction $(50.8 \mathrm{~g})$ was subjected to Sephadex LH-20 (MeOH) to give eight fractions (frs. F-M). Fraction $\mathrm{H}$ $(35.7 \mathrm{~g})$ was chromatographed over silica gel $\left[\mathrm{CHCl}_{3}-\mathrm{MeOH}-\mathrm{H}_{2} \mathrm{O}\right.$ $(4: 1: 0.1 \rightarrow 15: 5: 0.4 \rightarrow 7: 3: 0.5 \rightarrow 6: 4: 0.5 \rightarrow 1: 1: 0.1) \rightarrow \mathrm{MeOH}]$ to give ten fractions (frs. $\mathrm{H}_{1}-\mathrm{H}_{10}$ ). Fraction $\mathrm{H}_{5}(7.8 \mathrm{~g}$ ) was subjected to a Lobar RP-8 column $\left(\mathrm{H}_{2} \mathrm{O}\right)$ to give glycerol $(70 \mathrm{mg})$. Fraction $\mathrm{H}_{7}(7.8 \mathrm{~g})$ was subjected to a Lobar RP-8 column $\left(\mathrm{H}_{2} \mathrm{O}\right)$ and HPLC [CHA, MeCN- $\mathrm{H}_{2} \mathrm{O}$ $(14: 1)]$ to give D-hamamelose $(97 \mathrm{mg})$, D-fructose $(1.4 \mathrm{~g})$ and D-glucose $(1.1 \mathrm{~g})$. Fraction $\mathrm{H}_{8}(7.3 \mathrm{~g})$ was subjected to a Lobar RP-8 to give sucrose (182 mg).

The following compounds were identified by comparison of physical and NMR data with those reported: (Z)-3-hexenyl 1- $O$ - $\beta$-D-glucopyranoside (1), $(1 R, 2 R)-5^{\prime}$-hydroxyjasmonic acid $5^{\prime}-O-\beta$-D-glucopyranoside $(2),(1 R, 2 R)$ methyl-5'-hydroxyjasmonate $5^{\prime}-O$ - $\beta$-D-glucopyranoside $(3),(1 R, 2 R)-5^{\prime}$-hydroxyjasmonic acid $(\mathbf{5}),(1 R, 2 R)$-methyl-5'-hydroxyjasmonate (6), 3-hydroxy-4-methoxyphenethyl 3-O- $\beta$-D-glucopyranoside (7), eugenol $\beta$-D-glucopyranoside (8), syringin (9), and rosmarinic acid (10).

(Z)-5'-Hydroxyjasmone $5^{\prime}$ - $O$ - $\boldsymbol{\beta}$-D-Glucopyranoside (4) An amor- phous powder, $[\alpha]_{\mathrm{D}}^{22}-24^{\circ}(c=0.9, \mathrm{MeOH})$. Positive ion FAB-MS $m / z$ : $365.1562[\mathrm{M}+\mathrm{Na}]^{+}$(Calcd for $\left.\mathrm{C}_{17} \mathrm{H}_{26} \mathrm{NaO}_{7} ; 365.1576\right), 365.1562$ $[\mathrm{M}+\mathrm{Na}]^{+}\left(\mathrm{Calcd}\right.$ for $\left.\mathrm{C}_{17} \mathrm{H}_{26} \mathrm{NaO}_{7} ; 365.1576\right), 343.1754[\mathrm{M}+\mathrm{H}]^{+}$(Calcd for $\mathrm{C}_{17} \mathrm{H}_{27} \mathrm{O}_{7} ; 343.1757$ ), $163\left[\mathrm{M}-\mathrm{C}_{6} \mathrm{H}_{12} \mathrm{O}_{6}+\mathrm{H}\right]^{+}$(base). ${ }^{1} \mathrm{H}-\mathrm{NMR}$ (in pyridine$d_{5}$ ): 2.22 and 2.22 (each $2 \mathrm{H}$, ddd, $J=6.0,9.5,19.0 \mathrm{~Hz}, \mathrm{H}_{2}-4$ and $\mathrm{H}_{2}-5$ ), 3.00 $\left(2 \mathrm{H}\right.$, br d, $\left.J=7.0 \mathrm{~Hz}, \mathrm{H}_{2}-1^{\prime}\right), 5.48\left(1 \mathrm{H}, \mathrm{dt}, J=10.5,7.0 \mathrm{~Hz}, \mathrm{H}-2^{\prime}\right), 5.56(1 \mathrm{H}$, $\left.\mathrm{dt}, J=10.5,7.0 \mathrm{~Hz}, \mathrm{H}-3^{\prime}\right), 2.66\left(2 \mathrm{H}, \mathrm{dt}, J=7.0,7.0 \mathrm{~Hz}, \mathrm{H}_{2}-4^{\prime}\right), 3.78$ and 4.17 (each $1 \mathrm{H}, \mathrm{dt}, J=9.5,7.0 \mathrm{~Hz}, \mathrm{H}-5^{\prime} \mathrm{a}$ and $\mathrm{H}^{\prime} 5^{\prime} \mathrm{b}$ ), $1.84\left(3 \mathrm{H}, \mathrm{s}, \mathrm{H}_{3}-1^{\prime \prime}\right), 4.90$ $(1 \mathrm{H}, \mathrm{d}, J=8.0 \mathrm{~Hz}, \mathrm{Glc} \mathrm{H}-1), 4.07(1 \mathrm{H}$, br t $, J=8.0 \mathrm{~Hz}, \mathrm{Glc} \mathrm{H}-2), 4.29(1 \mathrm{H}$, brt, $J=8.0 \mathrm{~Hz}$, Glc H-3), $4.26(1 \mathrm{H}$, brt, $J=8.0 \mathrm{~Hz}, \mathrm{Glc} \mathrm{H}-4), 3.98(1 \mathrm{H}, \mathrm{m}$, Glc H-5), $4.40(1 \mathrm{H}, \mathrm{dd}, J=12.0,5.0 \mathrm{~Hz}$, Glc H-6a), $4.58(1 \mathrm{H}, \mathrm{dd}, J=12.0$, $2.0 \mathrm{~Hz}$, Glc H-6b). ${ }^{13} \mathrm{C}-\mathrm{NMR}$ (in pyridine- $d_{5}$ ): 170.33 (C-1), 138.88 (C-2), 208.15 (C-3), 34.41 (C-4), 31.57 (C-5), 21.79 (C-1'), 128.19 (C-2'), 126.81 (C-3'), 28.57 (C-4'), 69.28 (C-5'), 16.99 (C-1"), 104.71 (Glc C-1), 75.22 (Glc C-2), 78.60 (Glc C-3), 71.73 (Glc C-4), 78.54 (Glc C-5), 62.85 (Glc C6). HMBC Correlations: $\mathrm{H}_{2}-4 / \mathrm{C}-1, \mathrm{C}-2, \mathrm{C}-3, \mathrm{C}-5 ; \mathrm{H}_{2}-5 / \mathrm{C}-1, \mathrm{C}-2, \mathrm{C}-3, \mathrm{C}-4$; $\mathrm{H}_{2}-1^{\prime} / \mathrm{C}-1, \mathrm{C}-2, \mathrm{C}-3, \mathrm{C}-2^{\prime}, \mathrm{C}-3^{\prime} ; \mathrm{H}-2^{\prime} / \mathrm{C}-2, \mathrm{C}-1^{\prime}, \mathrm{C}-4^{\prime} ; \mathrm{H}-3^{\prime} / \mathrm{C}-1^{\prime}, \mathrm{C} 4^{\prime}, \mathrm{C}-5^{\prime}$; $\mathrm{H}-5^{\prime} \mathrm{a} / \mathrm{C}-3^{\prime}, \mathrm{C}-4^{\prime}$, Glc C-1; H-5'b/C-3', C-4', Glc C-1; $\mathrm{H}_{3}-1^{\prime \prime} / \mathrm{C}-1, \mathrm{C}-2, \mathrm{C}-5$; Glc $\mathrm{H}-1 / \mathrm{C}-5^{\prime}$.

Acid Hydrolysis of 4 Glucoside $4(3 \mathrm{mg})$ was heated at $95^{\circ} \mathrm{C}$ with with $5 \% \mathrm{H}_{2} \mathrm{SO}_{4}(2 \mathrm{ml})$ for $1 \mathrm{~h}$. After neutralization with Amberlite IRA-400 $\left(\mathrm{OH}^{-}\right.$form $)$, the reaction mixture was concentrated to give a sugar fraction. The sugar fraction was passed through Sephadex LH-20 $(\mathrm{MeOH})$ to give a syrup, and HPLC [carbohydrate analysis (Waters), detector; JASCO RI-930 detector, solv.; $\mathrm{MeCN}-\mathrm{H}_{2} \mathrm{O}(17: 3), 2 \mathrm{ml} / \mathrm{min} ; t_{\mathrm{R}} 4.50 \mathrm{~min}$ (same location as that of authenthic sample of D-glucose)] show the presence of glucose.

Acknowledgment The authors thank Mr. Takase Y., Dr. Suzuki H. and Miss Kiyotani T. of the Analytical Center of this University for NMR and MS measurements.

\section{References}

1) Wichtl M. (ed.), "Herbal Drugs and Phytopharmaceuticals," CRC Press, Stuttgart,1994, pp. 493-495.

2) Reddy M. V. B., Angers P., Gosselin A., Arul J., Phytochemistry, 47, $1515-1520$ (1998)

3) Rustaiyan A., Masoudi S., Monfared A., Kamalinejad M., Lajevardi T., Sedaghat S., Yari M., Planta Med., 66, 197-198 (2000).

4) Kitajima J., Ishikawa T., Satoh M., Phytochemistry, 64, 1003-1011 (2003).

5) Yoshihara T., Omer E. A., Koshino H., Sakamura S., Kikuta Y., Koda Y., Agric. Biol. Chem., 53, 2835-2837 (1989).

6) Gidda S. K., Misersch O., Levitin A., Schmidt J., Wasternack C., Varin L., J. Biol. Chem., 278, 17895-17900 (2003).

7) Kikuchi Y., Miyauchi Y., Chem. Pharm. Bull., 39, 1047-1050 (1991).

8) Orihara Y., Furuya T., Hashimoto N., Deguchi Y., Tokoro K., Kanisawa T., Phytochemistry, 31, 827-831 (1992).

9) Kitajima J., Ishikawa T., Tanaka Y., Ono M., Ito N., Nohara T., Chem. Pharm. Bull., 46, 1587-1590 (1998).

10) Kelly C. I., Mahajan J. R., Brooks L. C., Neubert L. A., Breneman W. R., Carmack M., J. Org. Chem., 40, 1804-1815 (1975).

11) Kelly C. I., Harruff R. C., Carmack M., J. Org. Chem., 41, 449-454 (1976).

12) Nagumo S., Kawai K., Nagase H., Inoue T., Nagai M., Yakugaku Zasshi, 104, 1223-1231 (1984).

13) Arimura G., Ozawa R., Horiuchi J., Nishioka T., Takabayashi J., Biochemical Systematics and Ecology, 29, 1049-1061 (2001).

14) Fujita T., Terato K., Nalayama M., Biosci. Biotech. Biochem., 60, 732-735 (1996)

15) Matsuura H., Yoshihara T., Ichihara A., Kikuta Y., Koda Y., Biosci. Biotec. Biochem., 57, 1253-1256 (1993).

16) Kasai R., Suzuo M., Asakawa J., Tanaka O., Tetrahedron Lett., 1977, 175-178 (1977)

17) Tori K., Seo S., Yoshimura Y., Arita Y., Tomita Y., Tetrahedron Lett., 1977, 179-182 (1977)

18) Klyne W., Biochem. J., 47, XIi-XIii (1950).

19) Miersch O., Schneider G., Sembdner G., Phytochemistry, 30, 40494051 (1991).

20) Kramell R., Schneider G., Miersch O., Phytochemical Analysis, 7, $209-212$ (1996). 